\title{
Spectral and temporal degradation of speech as a simulation of morphosyntactic deficits in English and German ${ }^{\text {is }}$
}

\author{
Frederic Dick, ${ }^{\mathrm{a}, *}$ Elizabeth Bates, ${ }^{\mathrm{a}}$ and Evelyn C. Ferstl ${ }^{\mathrm{b}}$ \\ ${ }^{a}$ Center for Research in Language and Department of Cognitive Science, University of California, 9500 Gilman Drive, Mail Code 0526, La Jolla, \\ San Diego, CA 92093-0526, USA \\ ${ }^{\mathrm{b}}$ Max-Planck-Institute for Cognitive Neuroscience, Leipzig, Germany
}

Accepted 21 March 2003

\begin{abstract}
Spectral and temporal degradation of the speech stream is increasingly used to model receptive language deficits such as aphasia and developmental language disorders. As with results from patient studies, the specific pattern of receptive deficits can reveal underlying structural and processing characteristics of different languages. Here, we test English- and German-speaking college students' auditory comprehension of complex morphosyntactic structures under normal and 'dual-degradation' conditions. The resulting profiles of strength and vulnerability in the two languages highlight the cross-linguistic differences in reliability of syntactic and morphological cues, and closely resemble the deficits observed in previous studies of receptive aphasia.
\end{abstract}

(C) 2003 Published by Elsevier Science (USA).

\section{Introduction}

Temporal and/or spectral degradation of the speech signal has been used for simulating or modeling a variety of language deficits, including those observed in aphasia (Miyake, Carpenter, \& Just, 1994; Moll, Cardillo, \& Aydelott Utman, 2001), Specific Language Impairment (Tallal, Stark, \& Mellits, 1985), and cognitive aging (Gordon-Salant \& Fitzgibbons, 2001). Converging evidence from several methodologies, such as ERP (Aydelott, Dick, \& Mills, 2002), fMRI (Davis \& Johnsrude, 2002; Poldrack et al., 2001), and neuropsy-

\footnotetext{
This research was supported by grants to Elizabeth Bates, "Cross-linguistic studies in aphasia" (NIH/NIDCD RO1-DC00216); to Frederic Dick "Language, Communication, and the Brain" CRL training grant (NIH T 32 DC00041), and "Training Program in Cognitive Neuroscience" NIH/NIMH 1 T32 MH20002-02. We thank the University of Leipzig and the Max-Planck-Institute for Cognitive Neuroscience for their support and the use of testing facilities; particular thanks go to Angela Friederici for invaluable assistance throughout. Finally, we are very grateful to our reviewers, whose comments substantially improved the paper, and whose patience with our questions was most appreciated.

${ }^{*}$ Corresponding author. Fax: +858-534-6788.

E-mail address: fdick@cogsci.ucsd.edu (F. Dick).
}

chology (Aydelott Utman, Blumstein, \& Sullivan, 2001; Moineau, Dronkers, Ludy, \& Bates, 2002) suggest that the brain regions and processes affected by such degradations overlap to a large extent with those affected in biologically based language breakdown.

These 'simulations' of language pathology also have been useful in testing models of speech and language comprehension, as well as in predicting the character and extent of deficits seen in patient populations. For instance, a recent study by Dick et al. (2001) directly compared syntactic comprehension by young adult native speakers of English under several degradation conditions with comprehension by several aphasic subgroups on a 'test of agrammatism.' In general, students under single degradations [speech compression, noise mask, low-pass filter] showed deficits qualitatively similar to those of aphasics (but were more accurate overall), while students under a particular 'dual-degradation' condition [low-pass filter plus compression] were qualitatively and quantitatively indistinguishable from the aphasic patients showing the classic 'agrammatic' profile of sentence comprehension.

Such speech degradation techniques can also be helpful when comparing the processing strategies and patterns of breakdown in languages whose means of 
conveying agency differ. Indeed, a large body of crosslinguistic studies using a competition model design (Bates, Wulfeck, \& MacWhinney, 1991) has shown that the 'same' cue to agency may have high informational value ('validity') in one language, and little to none in another. For instance, both English and German can convey sentential agency ('who done it') through word order and noun-verb number agreement cues; however, the value and determinacy of these cues differs across the two languages. In English, agency is unambiguously determined by the order of a sentence's constituents; in grammatical sentences, noun-verb agreement cannot clash with word-order-derived sentential roles, and only adds information when the grammatical number of agent and patient differ (e.g., 'The dogs are chasing the cat'). In contrast, noun-verb word order cues in German are 'soft' constraints (e.g., syntactically ambiguous), whereas noun-verb agreement information is unambiguous. A strong word order preference (such as Subject-Verb-Object (SVO) for Noun-Verb-Noun constructions) can be overridden by semantic role information, as well as by other syntactic cues, like noun-verb agreement. For instance, in German it is permissible to say 'the women (Frauen) feed (füttern) the goat (Ziege)' with either (1), 'Die Frauen füttern die Ziege' or (2), 'Die Ziege füttern die Frauen.' In phrase (1), the noun-verb agreement cues (underlined) converge with the SVO word order preference. In phrase (2), the noun-verb agreement conflict with the SVO preference, and force an OVS interpretation of the sentence. (We should note that the latter construction would be used in discourse situations where the sentence's focus was on the recipient of the action, such as 'Die Ziege füttern die Frauen, aber die Katze füttern die Männer,' literally 'The goat feed the women, but the cat feed the men,' or in standard English, 'The women feed the goat, but the men feed the cat.') Interestingly, relative to the 'soft' word order constraints, these deterministic $\mathrm{N}-\mathrm{V}$ agreement cues of German have proven to be particularly vulnerable to processing deficits in aphasia (Bates, Friederici, \& Wulfeck, 1987b).

Kilborn (1991) showed that these crosslinguistic patterns of strength and vulnerability found in aphasic patients also can be shown in neurologically intact speakers in normal and noisy listening conditions. In this study, German speakers listening to simple transitive sentences relied more on agreement and semantic cues than on word order, compared to English speakers. However, under noise conditions, German speakers' use of agreement cues was severely curtailed, while use of word order cues increased slightly, just as in previous studies of aphasia (Bates, Friederici, \& Wulfeck, 1987a, 1987b; Bates, Friederici, Wulfeck, \& Juarez, 1988). As predicted, English speakers paid little attention to $\mathrm{N}-\mathrm{V}$ agreement cues; their use of word order information was essentially unchanged in this single-degradation study.
However, as shown in Dick et al. (2001), single- and dual-degradation conditions can inhibit the use of English word order cues in more complex syntactic constructions, just as in aphasic patients. In order to test the relative strength and/or vulnerability of agreement and word order cues in languages where they are differentially weighted, here we will go beyond the original simulations of aphasia reported by Kilborn to test comprehension of the 4 more complex sentence types of Dick et al. (crossed with a noun-verb agreement cue factor) under normal and dual-degradation conditions. By using the most 'potent' combination of Dick et al.'s spectrotemporal degradations (namely a low-pass filter plus speech compression), we should be able to detect more subtle differences in subjects' processing strategies than would be possible with a simple degradation, such as a noise mask. In addition, we have deliberately designed our stimuli to take advantage of German's more flexible word order; this allows us to evaluate the robustness of noun-verb agreement when it is either a converging or conflicting cue to agency.

In English, we would expect that results would pattern as in Dick et al., where less frequent and hence less reliable word order strategies would be more vulnerable to degradation, just as in the original study; the presence or absence of the $\mathrm{N}-\mathrm{V}$ agreement cue should have minimal impact on processing in either condition. However, in German we should see effects of both cues to agency, with relatively strict reliance on agreement cues in the normal condition, and a greatly diminished ability to use these cues in dual-degradation conditions. Furthermore, we should see aphasic-like deficits arise in the use of less-preferred word order cues, such as the OVS order seen in passive sentences; however, the impact of the dual-degradation condition should be somewhat less on the word-order-driven sentence types than on the sentences where agency is determined by agreement cues.

\section{Method}

\subsection{Participants}

Fifty undergraduate students from the University of California, San Diego, and 47 students from the University of Leipzig, Germany took part in the study; San Diego students received class credit for their participation, while Leipzig students received monetary reimbursement. All participants were right-handed with normal hearing, and were native speakers of their language. All participants were treated in accordance with the "Ethical Principles of Psychologists and Code of Conduct" (American Psychological Association, 1992). 


\subsection{Design and materials}

The 2 within-subjects $\times 2$ between-subjects design was as follows: Within-subjects variables were Sentence Type (Active, Subject Cleft, Object Cleft, Passive) and Subject-Verb Agreement Cue (only subject agrees with verb in number, both nouns agree with verb). Between-subjects variables were Degradation Condition (Normal, $600 \mathrm{~Hz}$ Low-Pass Filter $+50 \%$ Speech Compression) and Language (English, German).

Visual stimuli were $3^{\prime \prime} \times 2^{\prime \prime}$ digitized black-and-white line drawings of familiar animals culled from several picture databases (Abbate \& LaChapelle, 1984a, 1984b; Snodgrass \& Vanderwart, 1980). Displayed on a VGA color monitor, each drawing was embedded in a solid gray rectangle over a white background; drawings were presented in pairs determined by sentence content, and projected to the left and right sides of the monitor.

Auditory sentence stimuli consisted of 96 sentences that were generated by first randomly assigning two animate nouns (from a pool of 12) to one transitive verb (from a pool of 8). All 12 nouns referred to familiar animals, and all could be assigned to either agent or patient roles. All nouns were of feminine grammatical gender and regular plural inflection in German in order to avoid confounds of case. All 8 verbs were semantically similar, in that they expressed a "bad action," such as killing or hurting, in order to highlight the asymmetry between actor and patient and thereby facilitate agent choice. All verbs were also selected so that, in German, they would not contain a separable prefix (which would create additional differences in structure between English and German equivalents). In English, the present progressive form of the verb was used for all sentences for the purposes of continuity with related studies such as Dick et al. (2001); in German, the simple present tense was used.

24 noun-verb pairs were then randomly assigned to each of the following four syntactic structures: Actives, Subject Clefts, Object Clefts, and Passives. Each of these pairs was then pseudorandomly assigned to one of four inflectional paradigms: (1) subject and object inflected in singular, verb agrees with both; (2) subject singular, object plural, verb agrees with subject; (3) subject plural, object singular, verb agrees with subject; (4) both subject and object plural, verb agrees with both. Each level of the Sentence Type variable was thereby represented by 24 exemplars, half of which contained a cue to agency via subject-verb agreement (inflections (2) and (3)), and half of which contained no agreement cue to agency (inflections (1) and (4)) - see Table 1 for example sentences.

Sentence stimuli were digitally recorded in a soundinsulated chamber by a native English- and a native German-speaking female (both without strong regional accents), and were normalized for speed, length (within sentence type), and amplitude. The lengths of the German stimuli were matched as closely as possible to the equivalent English stimuli; when numbers of syllables differed significantly, syllables per second was used as a matching guide. Noun stimuli (see below) were read one at a time. Recordings were then converted to SoundEdit16 files, with a $22.025 \mathrm{kHz}$ sampling rate and 16-bit quantization. The stimuli for the Low-Pass Filter + Speech Compression condition were generated in the same manner as in Dick et al. (2001); using proprietary SoundEdit16 algorithms, sound files were first compressed to $50 \%$ of their original length by excising redundant waveform information, thus preserving spectral integrity to a high degree. A $600 \mathrm{~Hz}$ low-pass filter was then applied to each shortened stimulus, reducing all spectral information above $600 \mathrm{~Hz}$ by $40 \mathrm{~dB}$. A subset of the altered sounds was inspected in both waveform and spectrogram format to verify the integrity of the phonemes signaling the agreement cues.

We further tested the intelligibility of noun-verb agreement cues in a transcription task. The task was as follows: An experiment-naïe native German speaker was asked to transcribe each sentence he heard as accurately as possible (but without worrying about capi-

Table 1

Examples of sentence types with and without disambiguating noun-verb agreement cues

\begin{tabular}{|c|c|c|}
\hline $\begin{array}{l}\text { Sentence type and presence/absence of } \\
\text { Agreement Cue (Agr/NoAgr) }\end{array}$ & English example sentence & German example sentence \\
\hline Active, NoAgr & The cat- is biting the goat- & Die Katze beisst die Ziege \# \\
\hline Active, Agr & The cats are biting the goat- & Die Katze $\underline{n}$ beissen die Ziege \\
\hline Subject Cleft, NoAgr & It's the cat- that is biting the goat- & Es ist die Katze \\
\hline Subject Cleft, Agr & It's the cats that are biting the goat- & Es sind die Katzen, die die Ziege beissen \\
\hline Object Cleft, NoAgr & It's the goat- that the cat- is biting & Es ist die Ziege-, die die Katze- beisst \# \\
\hline Object Cleft, Agr & It's the goat- that the cats are biting & Es ist die Ziege, die die Katzen beissen. \\
\hline Passive, NoAgr & The goat- is bitten by the cat- & Die Ziege wird von der Katze gebissen \\
\hline Passive, Agr & 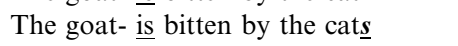 & Die Ziege wird von den Katzen gebissen \\
\hline
\end{tabular}

Inflectional cues for singular number are underlined (the zero form in English is marked with a hyphen). Cues for plural number are underlined, bolded, and in italics. Sentences that are syntactically and morphologically ambiguous in German are marked with a pound sign. Note that the Subject Cleft and Object Cleft without agreement cue are identical in form. 
talization or punctuation). He was given the same information about the sentences as was provided to the experimental participants-namely that each sentence described an animal or animals doing bad actions to other animals - and was blind to the underlying purpose of the transcription. As in the experiment, the transcriber heard (and transcribed) the 8 practice sentences in normal auditory presentation, then heard the same 8 sentences in LPC presentation. Also as in the experiment, the names of both agent and patient animal(s) were presented (in this case, read) to the transcriber in randomized order, followed by computer presentation of the sentence in LPC form. The transcriber was allowed to listen to each sentence multiple times in order to assure accuracy.

The transcriber made very few errors in inflectional morphology ( 7 out of a possible 384 errors); these errors occurred in 5 sentences, which were evenly distributed over sentence type (1 subject cleft, 2 passives, 1 object cleft, 1 simple active). A full list of errors as well as example sound files are available at http://crl.ucsd.edu/ $\sim$ fdick/B\&L.html. These results provide assurance that morphological cues were perceivable under stress, even though they might nevertheless prove difficult to use in real time.

\subsection{Equipment}

Psyscope software (version 1.0.1 and version 1.0.2) was used to deliver stimuli and collect data (Cohen, MacWhinney, Flatt, \& Provost, 1993). Software was run on Macintosh Performa 6214 computers, connected to a VGA color monitor, Apple external speakers, and Optimus headphones. A Psyscope button box was used for response and experimental timing.

\subsection{Procedure}

Participants were randomly assigned to Normal Listening or Low-Pass Filter plus Speech Compression (LPC) conditions (German Normal, $N=22$; German LPC, $N=25$; English Normal, $N=25$; English LPC, $N=25$ ). All were seated in a small room in front of a color monitor, speakers, and a Psyscope button box. Experimenters read instructions to the participants before the practice and experimental blocks; the practice block was composed of 8 trials, with the following experimental block composed of 96 trials. ${ }^{1}$ A trial consisted of the following: drawings of the animal agent(s)

\footnotetext{
${ }^{1}$ The entire experimental session consisted of two separate studies including the one presented here; the ordering of the studies was strictly counterbalanced to preclude systematic practice or fatigue effects. All sessions also began with a short baseline condition; the results of both these studies have been previously reported (Dick, Bates, Ferstl, \& Friederici, 1999a).
}

and patient(s) (e.g., 'giraffes,' 'goat') were projected on the left and right sides of the monitor over a gray background. (For the plural version, two identical drawings were put side by side). The animals' names were heard in succession, always in undistorted (nonLPC) form and randomly ordered. Participants then heard a sentence in either normal or LPC form, depending on the experimental condition assigned. Participants were instructed to use their right index finger in order to press the button corresponding to the picture of the animal(s) doing the bad action; the picture chosen by the participant was briefly highlighted before the screen was reset for the next trial.

Order of visual and auditory stimuli presentation was fully randomized for each participant, as was presentation of trials. Accuracy feedback was not provided. It was emphasized that participants should attempt to respond as accurately and quickly as possible to the stimuli. However, the experimenter also noted that some of the sentences could seem 'strange' or 'funny' and that, if the participant were unsure, s/he should respond with his or her best guess.

\section{Results}

The overall design of the experiment is a 2 (Language $) \times 2$ (Presence/Absence of Degradation $) \times 2$ (Presence/Absence of Converging Agreement) $\times 4$ (Sentence Type) where Language and Degradation Condition are between-subject variables and Agreement and Sentence Type are within-subjects. For all analyses involving within-subjects factors, reported $p$-values are Geisser-Greenhouse corrected; we report only those results falling at $p \leqslant .05$. In our prior studies of English (Dick et al., 2001), the dependent variable was 'percent correct.' This is not appropriate for the English-German comparisons, because some of the cells without agreement cues are ambiguous in German (that is, two readings are possible although there are preferred interpretations). Hence we will use "percent choice of the first noun as agent" (\%CFN) as our dependent variable, in line with earlier cross-linguistic studies by MacWhinney, Bates \& colleagues (Bates et al., 1991). ${ }^{2}$ For reaction time (RT) analyses, we calculate mean RTs from all responses except when specifically stated; RTs are based on reaction time post-sentence-offset. Because the thrust of most aphasia studies has been on accuracy or noun choice, we include here figures for $\% \mathrm{CFN}$ only, along with a table (Table 2) presenting pairwise com-

\footnotetext{
${ }^{2}$ We should note that the use of percent choice first noun does not affect the statistical significance of our results; when 'percent correct' was used as the dependent variable, $F$ - and $p$-values for all main effects and interactions were virtually identical to those from the percent choice first noun analyses.
} 
Table 2

Significance values for post-hoc pairwise contrasts between \%CFN means for sentences with and without agreement cues, split by sentence type, listening condition, and language

\begin{tabular}{lll}
\hline $\begin{array}{l}\text { Sentence type, } \\
\text { Listening condition }\end{array}$ & $\begin{array}{l}\text { English } \\
\text { Bonf./Raw }\end{array}$ & $\begin{array}{l}\text { German } \\
\text { Bonf./Raw }\end{array}$ \\
\hline Active, Normal & $--/--$ & $--/ .0246$ \\
Active, LPC & $--/ .0498$ & $.0176 / .0044$ \\
Subject Cleft, Normal & $--/--$ & $.0004 / .0001$ \\
Subject Cleft, LPC & $--/--$ & $--/--$ \\
Object Cleft, Normal & $--/--$ & $.0004 / .0001$ \\
Object Cleft, LPC & $--/--$ & $.014 / .0035$ \\
Passive, Normal & $--/--$ & $--/(.0726)$ \\
Passive, LPC & $--/ .0300$ & $--/--$ \\
\hline
\end{tabular}

Bonf./Raw, Bonferroni-corrected $p$-values (corrected for multiple comparisons within each language and condition)/Raw $p$-values uncorrected for multiple comparisons. Double-dashes (- -) signify that probability is greater than 0.05 , parentheses indicate marginally significant results. Significance values in italics indicate that the mean with agreement cue is lower than the mean without agreement cue.

parisons for each sentence type with and without agreement cue, separated by listening condition; figures with RT data can be seen at http://crl.ucsd.edu/ B\&L.html. The website also shows ANOVA tables for all analyses. We first present the omnibus ANOVA across languages (which is expected to result in a host of complex interactions, given the differences between these two languages), and then unpack these results in separate analyses for each language, with and without the disambiguating agreement cue.

\subsection{Omnibus analyses across languages}

For \%CFN, all of the main effects (Language, Degradation Condition, Sentence Type, Agreement) and all but one interaction (Language $\times$ Degradation Condition) reached significance at or fell below the GeisserGreenhouse (GG)-corrected $p \leqslant .05$ threshold. (The GG-corrected Sentence Type $\times$ Language $\times$ Condition interaction was on the margin of this threshold, at $p=.0538$ ) The four-way interaction is illustrated in Fig. 1. For RTs, all main effects were significant; all two- and three-way interactions involving Sentence Type were significant, while for Agreement, only the Agreement $\times$ Language $\times$ Condition interaction fell below threshold.

\subsection{Analysis for English only}

This analysis represents a replication of the same conditions for English in Dick et al. (2001), with a new set of participants, a different set of stimuli more closely matched to the constraints required for German, and a switch from accuracy to first-noun choice as the dependent variable. For Percent Choice First Noun $(\% \mathrm{CFN})$ the main effects of degradation condition and sentence type and the interaction between sentence type and condition were significant at $p=.0001$; these effects can be observed within Fig. 1. The overall result is in line with those of Dick et al. (2001): passives and object clefts were harder (with responses closer to the 50\% chance baseline) than actives and subject clefts, and degradation had a particularly severe effect on passives and object clefts (pushing them upward away from the 'correct' second-noun choice, and toward the chance baseline). Like Dick et al. (2001), none of the main effects or interactions involving agreement reached the $p<.05$ threshold. ${ }^{3}$ However, unlike the results of Dick et al. (2001), we did not observe a statistically significant advantage for passives versus object clefts in the dualdegradation condition.

RT results mirror the \% $\mathrm{CFN}$ effects of degradation condition, sentence type, and their interaction. Here, LPC slows subjects response times considerably, and RTs scale with sentence difficulty (actives $<$ subject clefts $<$ passives $<$ object clefts, with all means significantly different from each other); the Sentence Type $\times$ Condition interaction shows a small amplification of these sentence type effects in the LPC condition vs. normal listening. Interestingly, there was a small but significant RT advantage for sentences with agreement cues $(\sim 30 \mathrm{msec})$; however, this did not significantly interact with sentence type or degradation condition.

\subsection{Analysis for German only}

Compared to English, the results for German are more complex, particularly in terms of agreement (see Fig. 1). For $\% \mathrm{CFN}$, all main effects (Sentence Type, Agreement, Degradation Condition) and 2-way interactions between these factors were significant at the $p \leqslant .0002$ level; the 3-way interaction of all variables was significant at $p=.0001$. In general, converging information from agreement pushed performance in the preferred direction (first-noun choice for actives and subject clefts; second-noun choice for passives and object clefts). As expected, there is substantial ambiguity in the absence of agreement (keeping in that these sentences were intentionally designed to be ambiguous for

\footnotetext{
${ }^{3}$ A marginal interaction of Sentence Type $\times$ Agreement and Sentence Type $\times$ Agreement $\times$ Stress Condition (both at $p=.08$, GG-corrected) appears to be driven by the difference in passive performance with and without agreement, where disambiguating agreement information pulls scores closer to the chance $(50 \%)$ baseline, particularly in the dual-degradation condition. This marginal effect may be due to the 'reversed' role of the agreement cue in passives, where the verb agrees with the sentential patient rather than the agent - a result we have observed in other studies (Bates, Devescovi, \& Wulfeck, 2001; Dick, Wulfeck, Bates, Naucler, \& Dronkers, 1999b). The small and marginally significant drop in accuracy on actives with agreement cues in the LPC condition is not consistent with our previous findings.
} 


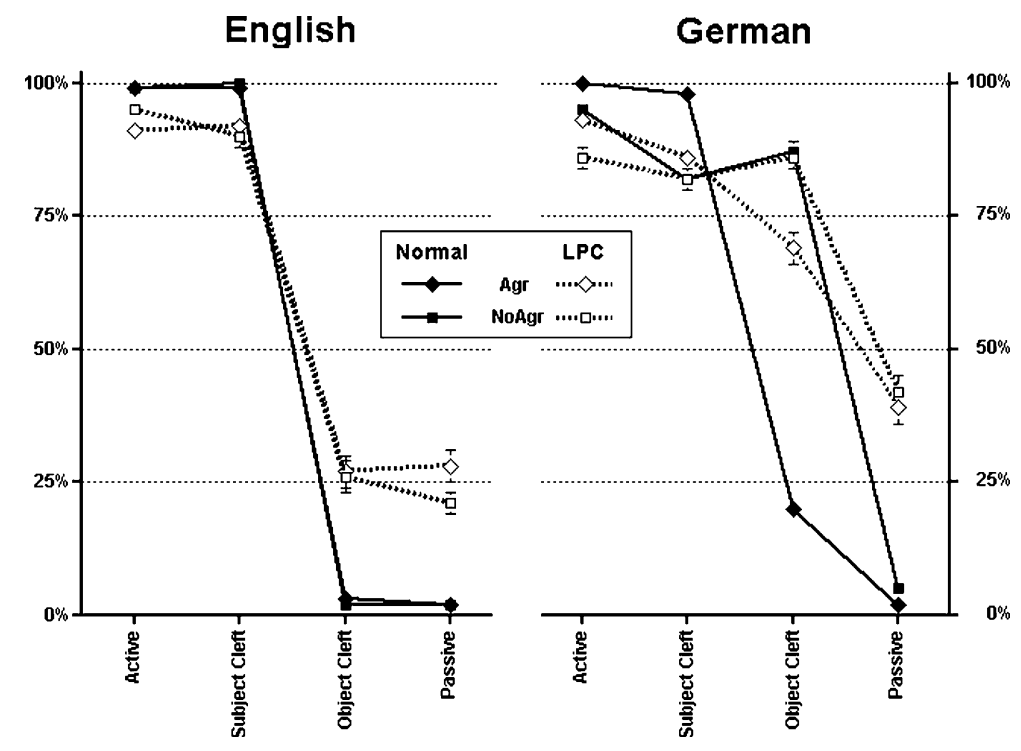

Fig. 1. English- and German-speakers performance under normal and dual-degradation conditions. Dependent measure is percent choice of first noun; error bars are $+/-1$ standard error of the individual cell mean. Abbreviations: LPC, Low-Pass Filter plus Speech Compression condition; Normal, Normal Listening; NoAgr, Absence of Disambiguating Noun-Verb Agreement Information; Agr, Presence of Disambiguating Noun-Verb Agreement Information; SubCleft, Subject Cleft; ObCleft, Object Cleft.

case, another important cue in German). In fact, the strings that were intended to serve as agreement-free controls for object clefts and subject clefts are actually indistinguishable for German listeners, regardless of degradation condition: in both cells, SOV was the preferred interpretation, reflected in $82 \%$ first-noun choice for agreement-free equivalents of subject clefts and $87 \%$ first-noun choice for agreement-free equivalents of object clefts (with both means having a relatively wide variance). Even in the presence of a disambiguating agreement cue for object-clefts, which is supposed to force a second-noun interpretation, first-noun choice averaged around $20 \%$ in the normal listening condition. Furthermore, some participants reported that they found these OSV object cleft items particularly strange, even though these are (in principle) grammatical items in German.

As with the English results, RT data tended to mirror $\%$ CFN data. Not surprisingly, RTs in the LPC condition were markedly slower than in normal listening. For all sentence types but object clefts, agreement cues in normal listening conditions allowed for a small reduction in reaction times, whereas reaction times for agreement-cued object clefts were the longest for all sentence types. However, RT reductions with agreement were essentially wiped out in the LPC version. Sentence Type effects reflected the classic increase in difficulty with non-canonical orders (with object clefts showing the slowest RTs) but also showed effects of ambiguity, with RTs to subject clefts as slow or slower than passives-actives were reacted to fastest. Passives were especially affected by degradation, with reaction times equal to those for agreement-signaled object clefts in the
LPC condition, but well below object cleft RTs in normal listening.

Overall, degradation hugely reduced the effectiveness of agreement cues, both in the case of converging agreement/word order cues (actives, subject clefts), and in the case of diverging cues (object clefts). But as in English, spectrotemporal degradation also greatly hindered comprehension of passives, which rely on a coalition of a non-canonical word order cue and the "byphrase' to convey agency.

\section{Discussion}

In line with previous cross-linguistic studies of sentence interpretation in English and German, we find that agreement plays a more important role for German listeners than it does for their English counterparts. In English, actives and passives interpretations are signaled unambiguously by passive morphology ("is eaten by...”), while subject- and object-cleft interpretations are signaled unambiguously by word order (SVO in for subject clefts; OSV for object clefts). Our English listeners rely heavily on these cues, and do not seem to make much use of converging information from agreement.

In contrast, agreement cues have a real impact for our German listeners, significantly increasing the proportion of 'preferred' interpretations for all four sentence types. The fact that agreement plays a larger role in comprehension of active sentences in German than in English is not particularly surprising, given that (unlike English) a German Noun-Verb-Noun sentence without passive 
morphology can be interpreted either as SVO or OVS even though the SVO interpretation is more common and greatly preferred in the absence of conflicting morphological cues (from case, agreement, or passive morphology). These results are entirely in tune with studies in other languages with relatively free word order, such as Spanish and Italian (Bates et al., 2001).

But the impact of the deterministic noun-verb agreement cue depends crucially upon whether it converges or conflicts with the powerful (but probabilistic) 'subject-first' word order preference. Given the identical word and constituent order (Noun/Noun/Verb), subjects in normal listening conditions will choose the agent signaled by noun-verb agreement $99 \%$ of the time (in subject clefts, where agreement and word order preference converge), or will follow agreement only $80 \%$ of the time (in object clefts, where agreement and word order conflict). When agreement cues converge with the lesspreferred passive word order (OSV) and accompanying deterministic morphological cues, they confer a small (and statistically marginal) advantage in choosing the syntactically correct interpretation in normal listening conditions. This last result suggests that the importance of noun-verb agreement for German listeners is not restricted to cases where 'soft' word-order preferences allow for a degree of ambiguity in the assignment of thematic roles.

Against this background, we have seen that the effects of perceptual degradation are quite distinct in English and German. In both languages, degradation drives performance toward (but not to) the chance baseline, and in both languages, effects are greater for the sentence types with non-canonical or non-preferred word orders (passives and object clefts). However, these effects interact in important ways with structural ambiguity and with the presence or absence of agreement information. Agreement matters very little in English; its use is neither increased nor decreased significantly by perceptual degradation, at least not in this experiment-for evidence that agreement morphology can be diminished by degradation in English, see Bates et al. (1994), Blackwell and Bates (1995), Blackwell, Bates, and Fisher (1996), and Kilborn (1991). (Indeed, in the LPC condition, agreement cues slightly reduced accuracy for passives and actives.) ${ }^{3}$ Instead, the overall effects of degradation for English revolve around the reduced interpretability of passives and object clefts. In German, the important disambiguating effects of agreement cues are dramatically diminished under degradation, particularly affecting interpretation of the agreement-cued object clefts. The non-preferred word-order cue used by passives is also hard hit, at least as much as in English, if not more so.

The effect of these degradations would be uninteresting if they were removing all relevant agreement information from the speech signal due to the interaction of the degradation used with the physical properties of the morphological cues. For instance, if the low-pass filter simply removed the part of the speech signal corresponding to the morpheme signaling singular or plural number on the verb (which are quite vulnerable acoustically), our results would be trivial. Four lines of evidence speak against this possibility. First, a low-pass filter alone (or speech compression alone) can reduce the use of agreement, but does not drive it to chance levels. Second, and as noted in Methods, we carefully checked stimuli spectrograms for the integrity of morphemes before and after degradations were applied. Third, the singular and plural markers for German verbs (' $\mathrm{t}$ ' and 'n,' respectively) are differentially affected by low-pass filtering. The wide-bandwidth noise burst signaling the ' $t$ ' is necessarily considerably diminished by the low-pass filter (although certainly still present), while the format transitions signaling the 'n' fall almost entirely under the $600 \mathrm{~Hz}$ cutoff, with a negligible difference in energy and waveform between normal and manipulated stimuli. If the agreement comprehension deficits we observe here are a product of loss of low-level acoustic information, rather than being due to the imposition of a more global 'stress' that makes higher level access and processing of these cues more difficult, then we should see a large difference in the effect of our dual-degradation condition on use of the singular and plural markers, particularly in the case of the agreement-based object clefts. However, a pairwise comparison revealed no such difference; indeed, cell means were within a single percent. Finally, the results of the transcription task showed that the LPC manipulation does not make perception of agreement cues impossible, and that perception of these cues does not vary systematically with sentence type.

In conclusion, the results reported here are in line with those from independent studies of sentence interpretation in English- and German-speaking aphasic patients. They also fit with the general 'subject-first' strategy that has been shown for such patients in English, German, Italian, and Dutch, as well as for normal subjects working under perceptual or attentional loads (Strube, 1996; Vos, Gunter, Kolk, \& Mulder, 2001a; Vos, Gunter, Schriefers, \& Friederici, 2001b). The specific result for German object clefts is especially interesting in light of a current controversy on the nature and causes of receptive agrammatism (Friederici \& Gorrell, 1998; Grodzinsky, 2000). Based on results for English, it has been argued that aphasic patients (whose results we have simulated here) find it difficult to process non-canonical word order configurations. In English, the subject cleft follows the same high-frequency SVO order that is used in active sentences, while the object cleft follows a low-frequency OSV order. In German, the subject- and object-clefts both occur in the same NounNoun-Verb frame, with the distinction between SOV (the preferred interpretation) and OSV (the 
non-preferred interpretation) signaled entirely by morphological cues. We have seen the object clefts are selectively vulnerable to degradation in both these languages, even though object clefts are realized in very different ways in English and German. Hence it appears that vulnerability to degradation may reflect the frequency of an entire construction (morphology included), as well as the frequency of its component parts.

\section{References}

Aydelott Utman, J., Blumstein, S., \& Sullivan, K. (2001). Mapping from sound to meaning: Reduced lexical activation in Broca's aphasics. Brain and Language, 79(3), 444-472.

Aydelott, J., Dick, F., \& Mills, D. (2002). Effects of Acoustic Distortion and Semantic Context on N400s to Spoken Words. Manuscript in review.

Abbate, M. S., \& LaChapelle, N. B. (1984a). Pictures, please! A language supplement. Communication Skill Builders.

Abbate, M. S., \& LaChapelle, N. B. (1984b). Pictures, please! An articulation supplement. Communication Skill Builders.

American Psychological Association, A.P. (1992). Ethical principles of psychologists and code of conduct. American Psychologist, 47, pp. 1597-1611.

Bates, E., Devescovi, A., Dronkers, N., Pizzamiglio, L., Wulfeck, B., \& Hernandez, A., et al. (1994). Grammatical deficits in patients without agrammatism: Sentence interpretation under stress in English and Italian. Abstracts from the Academy of Aphasia 1994 Annual Meeting. Special issue, Brain and Language 47(3), pp. 400-402.

Bates, E., Devescovi, A., \& Wulfeck, B. (2001). Psycholinguistics: A cross-language perspective. Annual Review of Psychology, 52, 369-398.

Bates, E., Friederici, A., \& Wulfeck, B. (1987a). Comprehension in aphasia: A cross-linguistic study. Brain and Language, 32(1), 19-67.

Bates, E., Friederici, A., \& Wulfeck, B. (1987b). Grammatical morphology in aphasia: Evidence from three languages. Cortex, 23(4), 545-574.

Bates, E., Friederici, A., Wulfeck, B., \& Juarez, L. A. (1988). On the preservation of word order in aphasia: Cross-linguistic evidence. Brain and Language, 33(2), 264-323.

Bates, E., Wulfeck, B., \& MacWhinney, B. (1991). Cross-linguistic studies in aphasia: An overview. Brain and Language, 41(2), 123-148.

Blackwell, A., \& Bates, E. (1995). Inducing agrammatic profiles in normals: Evidence for the selective vulnerability of morphology under cognitive resource limitation. Journal of Cognitive Neuroscience, 7(2), 228-257.

Blackwell, A., Bates, E., \& Fisher, D. (1996). The time course of grammaticality judgement. Language \& Cognitive Processes, 11(4), 337-406.

Cohen, J., MacWhinney, B., Flatt, M., \& Provost, J. (1993). PsyScope: A new graphic interactive environment for designing psychology experiments. Behavioral Research Methods, Instruments, and Computers, 25, 257-271.

Davis, M., \& Johnsrude, I. (2002). Hierarchical processing in spoken language comprehension. Journal of Neuroscience, in press.
Dick, F., Bates, E., Wulfeck, B., Aydelott Utman, J., Dronkers, N., \& Gernsbacher, M. A. (2001). Language deficits, localization, and grammar: Evidence for a distributive model of language breakdown in aphasic patients and neurologically intact individuals. Psychological Review, 108(4), 759-788.

Dick, F., Bates, E., Ferstl, E., \& Friederici, A. (1999a). Receptive agrammatism in English- and German-speaking college students processing under stress. Journal of Cognitive Neuroscience, Supplement.

Dick, F., Wulfeck, B., Bates, E., Naucler, N., \& Dronkers, N. (1999b). Interpretation of complex syntax by aphasic adults and children with focal lesions or specific language impairment. Brain \& Language, 69(3), 335-337.

Friederici, A. D., \& Gorrell, P. (1998). Structural prominence and agrammatic theta-role assignment: A reconsideration of linear strategies. Brain and Language, 65(2), 253-275.

Gordon-Salant, S., \& Fitzgibbons, P. J. (2001). Sources of age-related recognition difficulty for time-compressed speech. Journal of Speech, Language, and Hearing Research, 44(4), 709-719.

Grodzinsky, Y. (2000). The neurology of syntax: Language use without Broca's area. Behavioral and Brain Sciences, 1(23), $1-71$.

Kilborn, K. (1991). Selective impairment of grammatical morphology due to induced stress in normal listeners: Implications for aphasia. Brain and Language, 41(2), 275-288.

Miyake, A., Carpenter, P. A., \& Just, M. A. (1994). A capacity approach to syntactic comprehension disorders: Making normal adults perform like aphasic patients. Cognitive Neuropsychology, 11(6), 671-717.

Moineau, S., Dronkers, N., Ludy, C., \& Bates, E. (2002). Pushing the limits of word comprehension in normal and aphasic listeners. Supplement to the Journal of Cognitive Neuroscience, 9, 15.

Moll, K., Cardillo, E., \& Aydelott Utman, J. (2001). Effects of competing speech on sentence-word priming: semantic, perceptual, and attentional factors. In Proceedings of the Twenty-third Annual Conference of the Cognitive Science Society (pp. 679-684). Mahweh, NJ: Lawrence Erlbaum Associates.

Poldrack, R. A., Temple, E., Protopapas, A., Nagarajan, S., Tallal, P., \& Merzenich, M., et al. (2001). Relations between the neural bases of dynamic auditory processing and phonological processing: Evidence from fMRI. Journal of Cognitive Neuroscience, 13(5), 687-697.

Snodgrass, J. G., \& Vanderwart, M. (1980). A standardized set of 260 pictures: Norms for name agreement, familiarity and visual complexity. Journal of Experimental Psychology: Human Learning and Memory, 10, 174-215.

Strube, G. (1996). Sprachverarbeitung und Arbeitsgedächtnis: Syntaktische Analyse als automatischer Prozeß [Language processing and working memory: Syntactical analysis as an automatic process]. In: H. Mandl (Ed.), DPGs-Kongreß band.

Tallal, P., Stark, R. E., \& Mellits, E. D. (1985). Identification of language-impaired children on the basis of rapid perception and production skills. Brain and Language, 25(2), 314-322.

Vos, S., Gunter, T., Kolk, H. H. J., \& Mulder, G. (2001a). Working memory constraints on syntactic processing: An electrophysiological investigation. Psychophysiology, 38(1), 41-63.

Vos, S., Gunter, T., Schriefers, H., \& Friederici, A. (2001b). Syntactic parsing and working memory: The effects of syntactic complexity, reading span, and concurrent load. Language and Cognitive Processes, 16(1), 65-103. 